\section{REMARKS ON ALOPECIA AREATA. ${ }^{1}$}

By PHINEAS S. ABRAHAM, M.A., M.D.DuB., B.Sc.Lond, F.R.C.S. InHL.,

PHYSICIAN TO THE WESTERN SKIN HOSPITAL, LONDON, ETC.

THE diagnosis of alopecia areata is a matter of no difficulty in the majority of cases; but it is not invariably easy to isolate it clinically and pathologically from certain other affections of the hairy parts. More than one distinct disease, in fact, may have alopecia areata as a prominent feature or symptom. A localised alopecia, too, may result from external pressure or friction, as on infants' heads from pressure of the pillow, on ladies' heads in the days of the "chignon," and on men's shins just above the boot, as was pointed out to me by Mr. Rickard Lloyd. The literature of the affection from the time of Willan and Bateman onwards is instructive from more than one point of view. As with many other diseases, we find that great authorities from time to time publish some observation, or supposed observation, and, proceeding from the particular to the universal, forthwith enunciate a general theory, under which, of course, all cases must be made to fall. Although certain cases, as shown by M. Brocq, are the result of a folliculitis or perifolliculitis, no prior pustulation is met with in the vast majority of them, as was formerly believed. Again, the general belief in the specific fungus "microsporon Audouini" as the cause of alopecia areata lasted for years, in consequence, no doubt, of the common tendency of our profession to accept without personal investigation or corroboration all that our great men may say. There are still persons who believe that all cases of alopecia areata are of parasitic origin, and others who hold that all cases are neuropathic; but accurate observers in all parts of the world are more and more admitting that, while some are undoubtedly neurotic, many cases can only be explained on a parasitic hypothesis. Since Mr. Hutchinson and Dr. Radcliffe Crocker published their novel view that the majority of the cases of alopecia are etiologically related to common ringworm of the scalp I have as far as possible investigated the histories of 137 cases of alopecia areata, and have examined microscopically a considerable number. In no case which did not show other evidence of the affection being really tinea tonsurans could a trichophyton be found, and for some time it appeared to me that in com. paratively few instances could a history of previous ringworm be obtained. The evidence as to this latter point seems, however, to be increasing, and the statistics now presented undoubtedly lend some support to Mr. Hutchinson's and Dr. Crocker's theory. It is apparent, indeed, that in no less than forty-four instances, or 32 per cent. of the cases, there had either been a previous history of ringworm in the patient or other members of the family, or persons more or less in contact with the patient had been at some time or other affected with that disease. [Dr. Abraham gave short notes of thirty-seven of these cases.] There are certainly cases, on the other hand, in which no connexion with ringworm can be traced, and I can relate one, at least, in which I know and can vouch for all the facts. The question of the possible contagiousness of alopecia areata is also illustrated by my figures. In twenty-seven of the 137 cases there is a clear history of other members of the family or intimate friends having been affected with alopecia; but some of them are of very doubtful value in proving contagion, for the individuals have been affected at very different periods of time-years sometimes interveningor after they had been long living apart. Instances are referred to of the occasional occurrence of epidemics of alopecia areata, as noted by Bateman, the French writers, by Hillier, and others. Quite recently a somewhat analogous series of cases which occurred in an orphanage came under my notice, which, in accordance with our preconceived notions, may be taken either to show that at times alopecia areata is a very contagious disease or that that affection and common ringworm of the scalp are practically due to the same cause. Four boys were brought to me at the Western Skin Hospital with bare patches on the scalp. The patches were quite smooth and white, with a few short hairs with atrophrc roots around the margin and some loose atrophic hairs outside; no fungus could be found. The history was as follows. $A$ boy fourteen years of age (the patient was shown at the

1 Abstract of a paper read before the West London Medico-Chirurgical Society on Nov. 3rd, 1893. meeting) had ringworm four years ago and was said to have been cured. Bald patches commenced to appear about a year since. He shortly afterwards entered the orphanage and was passed as having no contagious disease. Since his admission eighteen of the boys of a total of twenty-two in the establishment had developed similar bald patches, and in most of them the hair had grown again under the influence of stimulating applications. Daring the spring of this year two other boys in the house showed signs of common ringworm; and within the last few weeks another boy (this patient was also shown) had undoubted tinea tonsurans. Three weeks ago the matron informed me that she, too, "had caught the disease"; and I found on her head three small round patches of commencing alopecia, without broken or clavate hairs, but with many loose hairs around with atrophied roots. In this case, too, no trichophyton could be demonstrated. She remembered that on one occasion she was interrupted before washing her hands after attending to the boys' heads, and she thinks that she then. touched her head. There was slight itching before the hair began to fall. For a long time I have observed that. certain persons suffering from alopecia areata had been the subjects of extensive seborrhoea, and I agree with M. Broco in thinking that some cases may be the sequel to that affection. The case of a woman with multiple patches was a marked instance ; I found that several other members of her family had extensive seborrhoea. Comparatively few (only thirteen) of the 137 cases gave a history of neuralgia or previous neuroses, and in those who complained of severe headaches I have just as frequently found the short clavate. hairs as in the others. I am aware that this is not in accordance with the views generally expressed. One of my cases illustrates this point. This patient is the mother of nine children, and none of them have ever had ringworm or alopecia; nor does she know of anything of the kind among friends. One of her patches is very depressed and scarlike. and shows club-shaped hairs at the borders; a smaller patch shows evident folliculitis. She had been out of health for some years, and suffered much from severe headaches; there was also extensive seborrhoea.

With reference to treatment, parasiticidal ointments and the cautious occasional application of Burt's fluid are, I believe, serviceable in a majority of the cases. I have by these means seen alopecias cured which had lasted for years and were apparently hopeless. I frequently use a carbolic and salicylic acid ointment, and sometimes intermit with lotions like the "lotion excitante" of the St. Louis Hospital. I always treat the seborrbon if present. When I want to impregnate an area of scalp with a germicidal fluid I am now trying an apparatus made for me some months ago by Messrs. Maw, Son, and Thompson, which is constructed on the principle employed in creasoting logs of wood or railway sleepers. A partial vacuum is first produced over the diseased part, which has been previously epilated, shaved and washed with soft-soap, alcohol, and ether; the germicide is then let in under the influence of the atmospheric pressure. It is curious to obserre how the spots implicated come into view by a manifest localised hyperæmia of the subjacent cutis, a fact of some pathological significance. I have only been able to use this method in a few cases, and I can say but verm little at present as to results.

\section{Clinital hotes:}

\section{MEDICAL, SURGICAL, OBSTETRICAL, AND THERAPEUTICAL.}

\section{CASE OF FRACTURE OF THE BASE OF THE SKULL: RECOVERY.}

By David Livingstone Davies, M.R C.S.Eng., L.R.C.P. LOND.

SURGEON TO THE FESTINIOG QUARRIES ; LATE ASSISTANT MEDICAL OFFICER TO THE BOROUGH ASYLUM, NOTTINGHAM.

THE following case appears to be worthy of record, showing as it does the not altogether unfavourable prognosis of cases of fractured base occurring in elderly people.

A woman fifty-six years of age was sitting on the shore at the foot of some cliffs about 3 o'clock in the afternoon on Aug. 10th, 1893. Two little boys who were with her climbed up the cliffs to the height of about $40 \mathrm{ft}$., and in doing so one 
of them dislodged a stone of flint weighing nearly $15 \mathrm{lb}$; this came rolling down and struck the woman in an oblique direction on the cranium at a point three inches above the base of the mastoid process of the temporal bone on the right side, from there glancing to the acromial end of the clavicle on the same side. When I saw her a few minutes after the accident she was lying in an unconscious state on the sand, bleeding from the right ear, and vomiting a dark fluid, consisting mostly of swallowed blood. She was carefully removed to the nearest house and put to bed. She had all the signs of concussion, the body being cold, the pulse slow and feeble ( 40 per minute), the respiration slow and sighing, and the pupils contracted. A hæmatoma formed over the part struck, and a fracture extending downwards and across the base of the skull through the petrous portion of the temporal bone was diagnosed. A few hours after the accident I had a consultation with Mr. Hunter Hughes, who confirmed my diagnosis.

The treatment briefly consisted in keeping the room darkened and perfectly quiet, applying ice to the head, hotwater bottles to the feet, giving fluid nourishment in the form of meat juice, syringing the ear with weak carbolic lotion, and then blowing in some boric acid and iodoform. The bowels were kept regular with five-grain doses of calomel every other day. A little brandy and milk was given for the first few hours in order to stimulate the heart, which was very weak. During the last two weeks she was allowed a little light wine and took a mixture of citrate of iron and ammonia with compound spirit of ammonia for the anæmia which resulted from the loss of blood. It will, perhaps, be observed that the ear was not plugged in order to arrest the hæmorrhage. I believe that in treating a case of fractured base of the skull it is wiser to allow the blood to escape externally. Many of the books which I referred to advise plugging the ear in order to arrest the hæmorrhage. I feel sure that had I done so, as I was at first inclined to, the hæmorrhagic attacks which she had would have produced fatal compression.

The after-course was uneventful; the patient recovered consciousness in three hours, and vomiting ceased in about twelve hours. After recovering consciousness she complained of pain in a line downwards from the point struck, probably along the line of fracture. There was acute tenderness for about ten days along this line. She took nourishment well and after the first two or three nights slept well. She had attacks of hæmorrhage from the ear on several occasions during the first three weeks, and some cerebro-spinal fluid oozed for the first week. She complained very much of pain and inability to move the right shoulder, but nothing more than a severe contusion could be detected, and this was well in three weeks. Some days after the accident she complained of tasting blood in her mouth, which she said made her feel sick. On examination blood could be seen slowly trickling through the orifice of the Eustachian tube. The patient gradually regained strength, so that on Sept. 21st, nearly six weeks after the accident, she was able to undertake the journey home to London, a distance of more than 200 miles from here. I have recently heard from her that she feels well and has no pain or headache.

Preswylfa, Criccieth, North Wales.

\section{SCARLET FEVER AND FNTERIC FEVER.}

BY S. A. E. GRIFFITHS, M.R.C.S. ENG., L.R.C.P. LoND.

DURING an epidemic of scarlet fever at Irthlingborough I had four patients from one family who developed enteric fever at the same time as, or very shortly after, they were affected with scarlet fever. I thought that the co-existence of two acute diseases, such as scarlet fever and enteric fever, in the same patient at the same time was of sufficient rarity to warrant my recording it.

CASE 1.-A boy aged eleven years was notified by me as suffering from scarlet fever on June 26th. The fever apparently for the first three days ran a fairly normal course, but the boy appeared to be rather more dull and restless than is usual with an ordinary case of scarlet fever. On the fourth day (June 30 th) the temperature was $103^{\circ} \mathrm{F}$., having only varied a degree or so from the first. Instead of the temperature falling, there was a tendency for it to rise. I suspected that the boy had developed pneumonia. I carefully auscultated the chest, but detected nothing abnormal. At this time (the 30 th) the tongue had gone through the stages usually seen in a well-marked case of scarlet fever, from being covered with white fur to a dark-red glazed appearance. The next day (July 1st) the tongue was again coated with fur (slightly) and in the course of the next two days assumed the characteristic typhoid appearance. Diarrhoea set in, with typhoid stools. On July 3rd I re-notified the case as "enterica. previously affected with scarlatina." Desquamation had already commenced and was very marked. On July 4th the case was seen with me by Mr. Burland, who confirmed my diagnosis. On July 6 th sudamina appeared, more especially over the chest, abdomen, and thighs. The temperature continued to be rather high for the first fortnight, varying between $102^{\circ}$ and $104^{\circ}$. The enteric fever ran a fairly normal course; spots appeared on the abdomen and lower part of the chest; the spleen was not markedly enlarged. From July 3rd to the 10th the boy was very delirious, being with difficulty kept in bed; he suffered great pain in his head. I gave him antipyrin and had him sponged with cold water every hour, from which he derived great relief. Convalescence was much delayed by relapses. His sister, who developed enteric fever during his convalescence, was even more delirious than her brother. On two occasions he was very much frightened by the delirium of his sister, relapses occurring. One day the temperature ran up to $105^{\circ}$ from normal. The house was so small that it was impossible to have the patients in separate rooms.

CASE 2. - The sister of the above patient, aged thirteen years, was notified as suffering from scarlet fever on July 15th. It ran a typical course; the glands in the neck were much enlarged and suppurated on the right side. An incision was made, the abscess causing difficulty in breathing. She developed enteric fever on July 27th, which was notified. This patient had a very poor constitution and was very delirious. It was almost impossible to keep her in bed. When she was quiet the delirium was low and muttering. I gave her antipyrin and large quantities of brandy. The temperature varied between $102^{\circ}$ and $105^{\circ} \mathrm{F}$. Diarrhoea was severe at times. The abscess discharged the whole of the time up to her convalescence; it was impossible, owing to her state, to attend to it at times.

CASE 3 -Another sister, aged three, was notified as suffering from scarlet fever on July 19th. This child was much debilitated and suffered from the effects of acute poliomyelitis in infancy. She was much prostrated from the scarlet fever and developed enteric fever on July 30th, which was notified. The temperature was $105^{\circ} \mathrm{F}$. I ordered cold spongings every hour and antipyrin. Although the temperature fell a couple of degrees it again rose. The child died on the next day from exhaustion.

CASE 4-A third sister, aged seven, was notified with scarlet fever on July 19th and with enteric fever on Aug. 1st. This patient had both the fevers mildly.

Remarks. - In the first case the difficulty of diagnosing the second fever was great on account of the more acute symptoms of scarlet fever masking those of enteric ferer, and, although on the fourth day I felt sure in my own mind that there was a second febrile disease underlying the scarlet fever, yet I hesitated before re-notifying (for by this one's diagnosis is made) till the more acute symptoms of scarlet fever had passed off. In the four cases the longest interval between the two fevers was thirteen days, and it is curious that in the last three cases the period was nearly the same-viz., eleven, twelve, and thirteen days. This would agree with the incubation period of ten to fifteen days. It is quite possible that when each was affected the two "poisons" were combined and that the scarlatinal poison had almost or entirely run its course before the other showed itself. Be this as it may, there is no doubt that the enteric "poison" was in the system of each during the scarlatinal course. In the case of the boy the two fevers practically ran concurrently; this, according to THE LANCET of Nov. 4th (p. 1149), appears to be possible. It will be of interest to add that another member of this family was affected with scarlet fever only.

Irthlingborough.

\section{EXCEPTIONAL CASES OF SCARLET FEVER.} By Duncan J. Cadpy, M.B.

CAsE 1.-On May 30th of this year I was called to see a child aged two years and five months. The mother stated that six days previously she had taken it to see her sister's children, one of whom was ill in bed with scarlet fever, but 